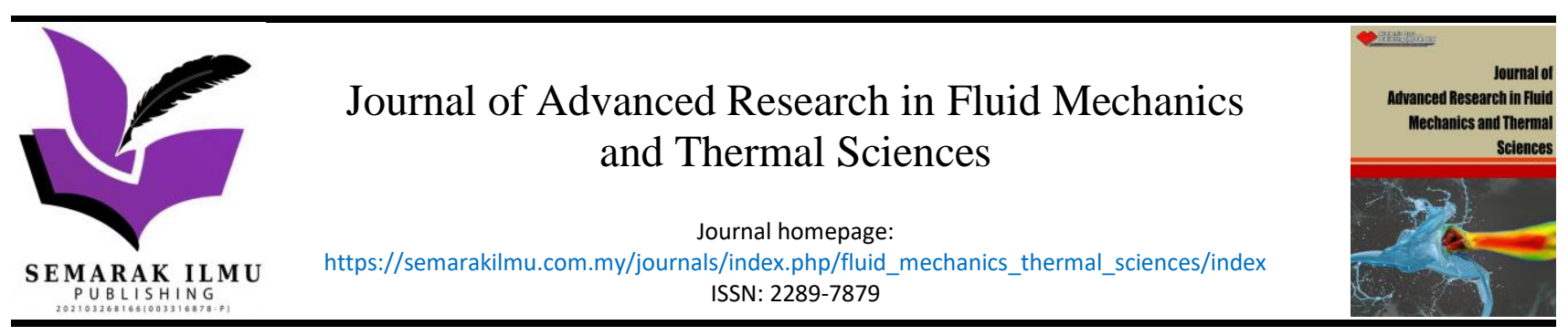

\title{
Development Of an Affordable Negative-Pressure Full-Body Isolation Pod for Covid-19 Patient Transportation
}

\author{
Muhammad Iftishah Ramdan ${ }^{1}$, Inzarulfaisham Abd Rahim ${ }^{1,}{ }^{*}$, Nik Hisamuddin Nik Ab Rahman ${ }^{2}$, \\ Ahmad Faizul Hawary ${ }^{3}$, Mohd Azmi Ismail ${ }^{1}$, Mark Selvan ${ }^{1}$, Lim Ban Aik ${ }^{1}$, Alexander Tan Wai Teng ${ }^{1}$, \\ Lim Wern Pink ${ }^{1}$
}

School of Mechanical Engineering, Engineering Campus, Universiti Sains Malaysia, 14300 Nibong Tebal, Penang, Malaysia

School of Medical Sciences, Health Campus, Universiti Sains Malaysia, Kota Bharu, 16150, Kelantan, Malaysia

School of Aerospace Engineering, Engineering Campus, Universiti Sains Malaysia, 14300 Nibong Tebal, Penang, Malaysia

\section{\begin{tabular}{l} 
ARTICLE INFO ABSTRACT \\
\hline
\end{tabular}}

\section{Article history:}

Received 2 August 2021

Received in revised form 15 October 2021

Accepted 20 October 2021

Available online 13 November 2021

Keywords:

Covid-19; healthcare workers; patient transportation

\begin{abstract}
Healthcare workers (HCWs) are known to be at a high risk of being exposed to the SARSCoV-2, or the COVID-19 virus because they are required to interact with their patients at a distance of less than two meters, for more than ten minutes at a time. Due to limited resources, most HCWs are inadequately protected. This study describes the development and the performance of an affordable full-body isolation pod (isopod). The isopod structure consists of composite side panels and aluminum poles and strips. A clear tarpaulin sheet is used to seal its structure. Two identical a high-efficiency particulate air (HEPA) filters are used to filter the air that goes through the isopod while the pressure inside it is maintained within the range described by the Airborne Infection Isolation (AII) room of the United Stated of America Centers for Disease Control and Prevention (CDC). This isopod could be the solution for hospitals in developing countries to enhance the protection among HCWs from the deadly virus since it is attainable and satisfies the CDC requirements.
\end{abstract}

\section{Introduction}

The Covid-19 pandemic has affected the lives of millions across the globe [1]. The Covid-19 virus has spread very efficiently and claimed more than four million lives worldwide thus far [2]. HCWs are part of the infected statistics because they are exposed to the virus via contact with infected patients [3-8]. Emergency HCWs are at an especially high risk of infection since they work in tight spaces, such as ambulances, forcing them to breathe the same air as the infected patients. Ideally, emergency HCWs should be protected by placing the patients in a negative-pressure isolation space [9-12]. In terms of public health, the health and the safety of the HCWs is extremely important, because the infected HCWs could be the spreaders of nosocomial infections [13-15].

\footnotetext{
* Corresponding author.

E-mail address: inzarul@usm.my
}

https://doi.org/10.37934/arfmts.88.3.137144 
This study proposes the use of an affordable portable negative-pressure full body isopod to create a sustainable barrier between HCWs and the infected patients during transportation. The isopod uses a blower inlet to create negative pressure, or vacuum, inside its chamber. The blower inlet is also equipped with HEPA filter, to ensure the air coming out of the isopod is virus-free. Since there is negative pressure inside the isopod, the ambient air is drawn in through another HEPA filter, allowing the ambient air intake to flow through the patient in the isopod. The negative pressure inside the isopod and the HEPA filters prevent contaminants from escaping the pod and affecting the medical staff caring for the patient.

The isopod can also be used in any clinical care environment, ranging from prehospital care transportation to intra-hospital transportation, and during emergency medical imaging, such as CT scanning and plain radiography, without disturbing the image quality. Furthermore, the isopod can also be used for transporting critically ill patients whose airways are protected by endotracheal intubation, thus markedly reducing the risk of airborne particles being transmitted to healthcare providers and ensuring their safety.

The current market value of imported isopods is estimated at USD 8,000 or MYR 32,000 while the one that is used in this study costs one-fifth of that. The low cost is achieved by utilizing parts that can be bought from hardware and electronic stores. The isopod also must satisfy the All room standard set by CDC [6]. This study tests the performance of the isopod to ensure it can produce and sustain the negative pressure set by CDC.

\section{Methodology}

\subsection{Design}

A full-body isopod is an important piece of personal protective equipment (PPE), designed to keep the emergency HCWs from getting infected. It is attachable to an ambulance stretcher and can be stored in a container when not needed. The detailed specifications of the isopod are shown in Table 1.

Table 1

Specifications of the full-body isopod

\begin{tabular}{ll}
\hline Weight (kg) & 21.7 \\
\hline Dimensions (cm) & $198 \times 61 \times 30.5$ \\
Filter size (mm) & $135 \times 135 \times 69$ \\
Filter efficiency (\%) @ most penetrating particle size (MPPS) [15] & $>99.995$ \\
Filter efficiency (\%) @ $0.3 \mu \mathrm{m}[15]$ & $>99.999$ \\
Number of glove holes & 9 \\
Number of snorkel holes & 1 \\
\hline
\end{tabular}

The structure of the isopod consists of two fibre-glass sideboards, four aluminium strips, ten glove cases, a clear vinyl, a tarpaulin sheet and two aluminium poles (Figure 1). During its assembly, the isopod tarpaulin base is unfolded and laid out on a transfer stretcher or an ambulance stretcher. Then, two aluminium poles are attached to two sideboards as its base structure. Finally, the four aluminium strips are inserted into the clear vinyl top and the patient can be placed inside the isopod before the zipper is closed by the accompanying physician. This process should take approximately five minutes.

The isopod is predominantly clear vinyl, with minimal non-ferrous metal content in its structure. The size enables it to fit comfortably into a CT scan gantry, a plain radiography table, or an ambulance rear compartment. After use, the isopod can be wiped down with a dishwashing sponge soaked in a 
detergent, to remove visible stains. It can be soaked in a $0.1 \%$ sodium hypochlorite solution for one hour and rinsed with water, then air dried for reuse, following the hospital infectious disease prevention protocol. However, the use of isopods should be governed by local institutional protocols, suitability for individual patients, and adherence to safety precautions.

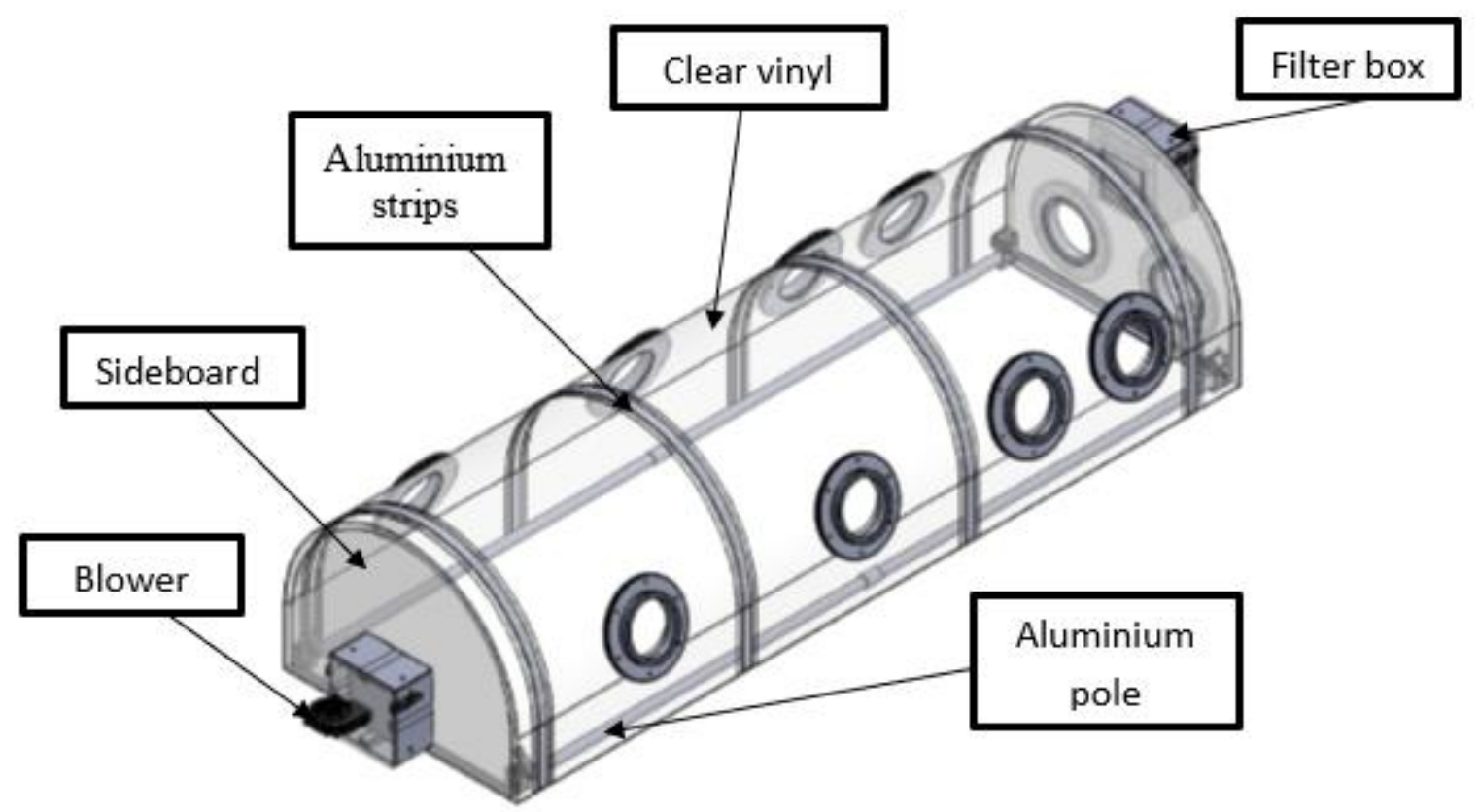

Fig. 1. Overall ISOPOD system

\subsection{Control of the blower}

The isopod filtering system consists of two filter boxes, one with a blower, located at the patient's head (Figure 2), and another, without a blower, located at the patient's feet. Both filter boxes are equipped with identical HEPA filters [16], oriented in the same direction as the air flow. The blower control system runs on a lithium-ion battery, which can last up to five hours. The isopod target pressure is set according to the All room requirement by CDC as stated in Table 2 in which the pressure should remain at least 2.5 Pa lower than atmospheric pressure [17].

Table 2

CDC requirement for All room

\begin{tabular}{ll}
\hline & Negative pressure mode \\
& All CDC requirements [16] \\
\hline Pressure differentials $(\mathrm{Pa})$ & $<-2.5 \mathrm{~Pa}$ \\
Ideal pressure differential $(\mathrm{Pa})$ & $<-2.5 \mathrm{~Pa}$ \\
Filtration Efficiency & Supply: $90 \%$ dust spot test \\
& Return: $99.97 \% @ 0.3$ um \\
& DOP1,3 \\
\hline
\end{tabular}




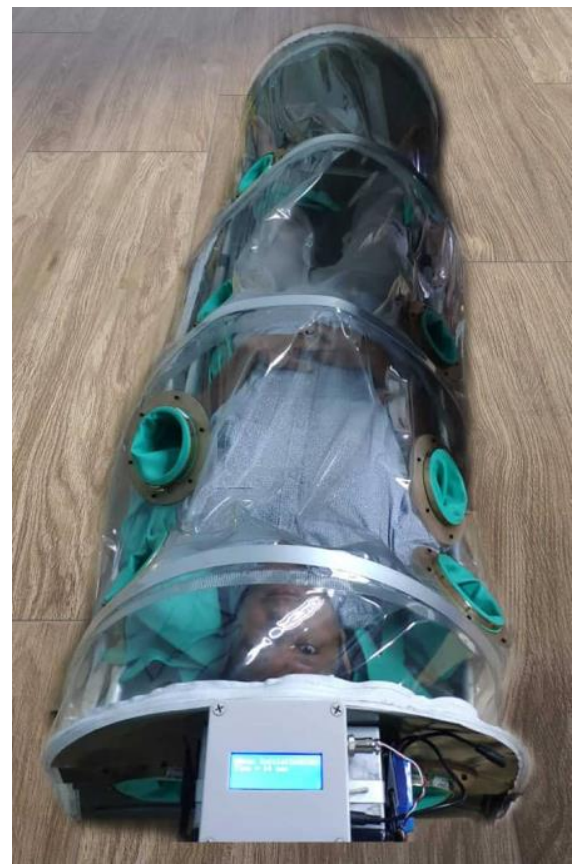

Fig. 2. Complete isopod

The blower speed is controlled by an Arduino-Nano micro controller that receives two signal inputs from pressure sensors. One pressure signal is from inside the chamber, while the other is from outside the chamber. The controller in Figure 3 and 4 adjusts the blower speed, according to the pressure difference between the inside and the outside the pod chamber, using a proportional integral derivative (PID) controller algorithm. This feature is needed to ensure the vacuum can be maintained even if the ambient pressure changes, due to a change in the altitude.

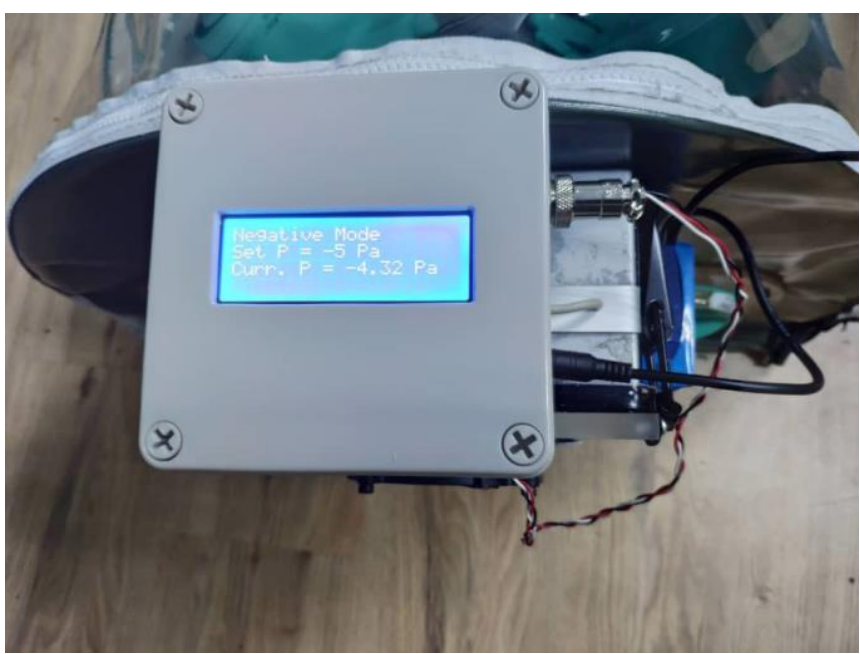

Fig. 3. The blower is running when the desired vacuum is not achieved 


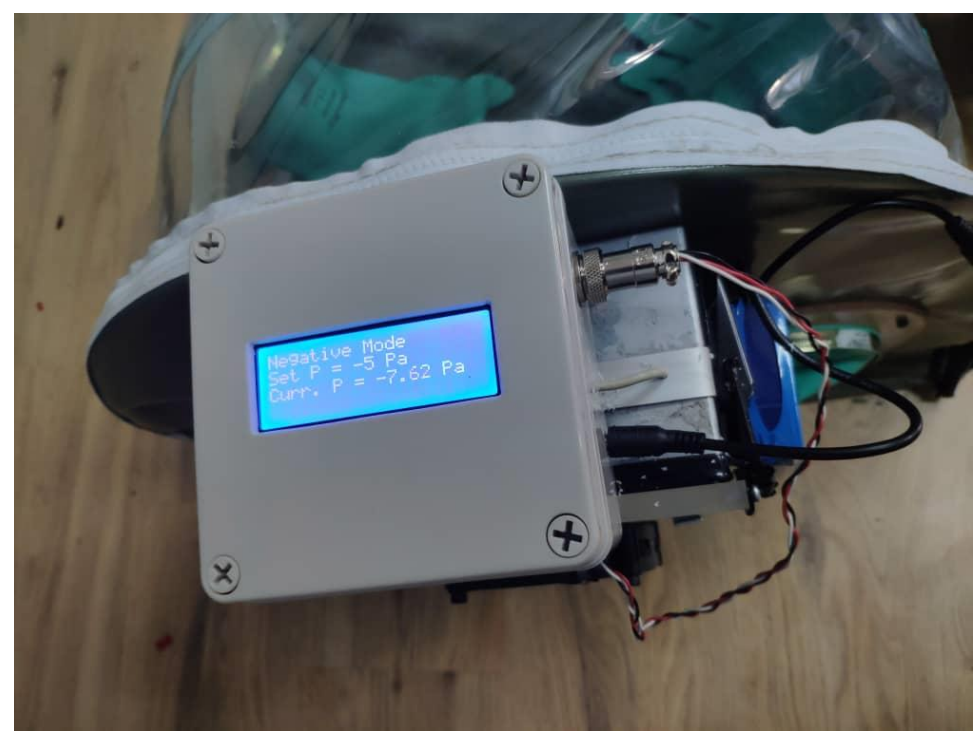

Fig. 4. The blower is stopped when there is vacuum overshoot

\section{Results}

The isopod blower speed controller is dependent on the pressure difference between the inside and the outside of the isopod. The target vacuum, or pressure difference between outside and inside, is set at $-5 \mathrm{~Pa}$. In this study, the pressure inside the chamber is measured in two locations (i.e., the head and the feet) every 30 seconds, to ensure the isopod can maintain the vacuum of at least -2.5 $\mathrm{Pa}$, as required by CDC. The pressure readings of the test can be seen in Table 3 .

\begin{tabular}{|c|c|c|}
\hline Time (s) & $\begin{array}{l}\text { Head-end } \\
\text { pressure (Pa) }\end{array}$ & $\begin{array}{l}\text { Feet-end } \\
\text { pressure }(\mathrm{Pa})\end{array}$ \\
\hline 0 & -5 & -6 \\
\hline 15 & -6 & -7 \\
\hline 30 & -10 & -7 \\
\hline 45 & -6 & -8 \\
\hline 60 & -9 & -10 \\
\hline 75 & -8 & -7 \\
\hline 590 & -10 & -8 \\
\hline 105 & -16 & -7 \\
\hline 120 & -10 & -8 \\
\hline 135 & -9 & -7 \\
\hline 150 & -12 & -8 \\
\hline 165 & -10 & -9 \\
\hline 180 & -17 & -9 \\
\hline
\end{tabular}

The pressure profiles at the two places follow similar trends, but the vacuum at the head (Figure 5 ) is stronger than that at the feet (Figure 6). A vacuum stronger than $-2.5 \mathrm{~Pa}$ is observed at both places and satisfies the All room requirement by CDC. However, both pressure values show a zig-zag trend (Figure 5 and 6). This is due to the controller activating the blower and creating stronger vacuum than the threshold, overshooting the target value (Figure 4). This causes the controller to reduce both the blower speed, and the vacuum strength until it drops below the threshold value again (Figure 3). This phenomenon causes the blower speed to oscillate between fast and slow. 


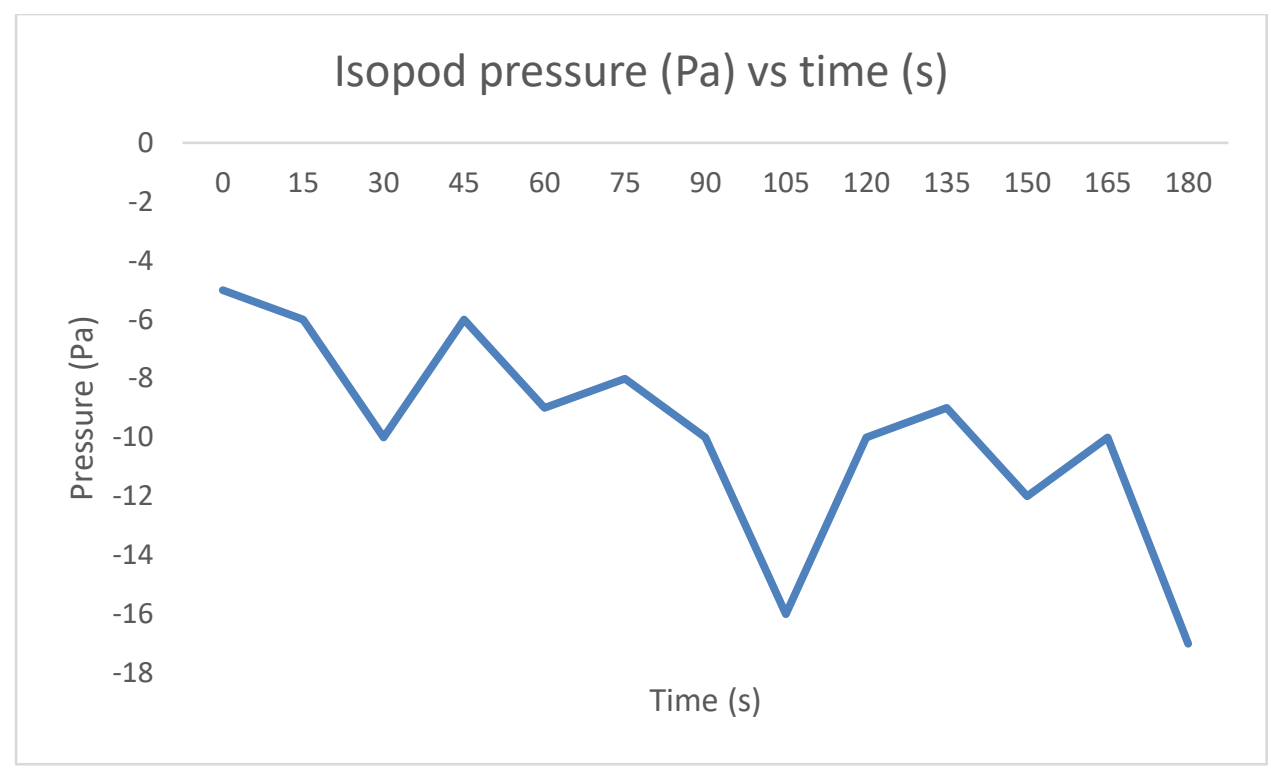

Fig. 5. Head-end pressure vs time

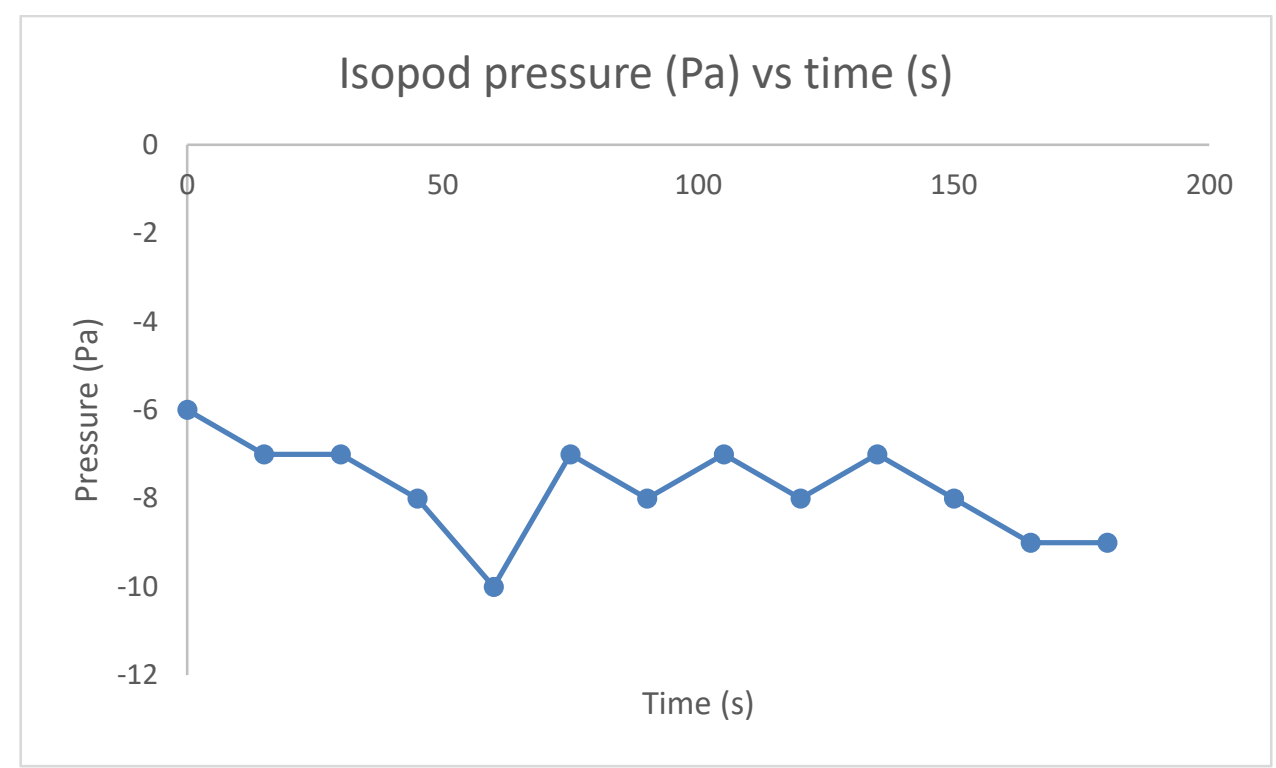

Fig. 6. Feet-end pressure vs time

\section{Discussion}

The attempt by our team to create an affordable ISOPOD product, made using readily available resources whilst maintaining the specification requirements of its function, based on the CDC recommendations, has proven successful. Furthermore, we have proven that the innovation reduces the costs, due to its price being a fifth of the current market value. The isopod will have a significant health and economic impact in the already financially constrained settings, especially in developing and low-income countries. Equally important is the innovation maintaining its clinical functionality, comparable to other marketed products.

An important safety feature was incorporated into the product, where two pressure sensors were installed to monitor both pressure values, one inside the isopod chamber, and one outside the chamber. These values serve as input to the micro-controller, which then adjusts the blower speed accordingly, in order to maintain the correct range of vacuum inside the chamber. The isopod also features nine glove penetrators on the isopod main frame, enabling the health care providers to 
conduct physical examinations and procedures without unzipping the isopod, thus maintaining the staff safety.

\section{Conclusions}

As the number of Covid-19 cases continues to increase, a full-body isopod is a very important piece of the PPE, protecting the emergency HCWs from the virus. This study describes the construction and the performance of an affordable isopod. The study shows that the isopod manages to satisfy the IIP requirement to maintain the pressure of at least 2.5 Pa below atmosphere.

\section{Acknowledgement}

We would like to thank the Universiti Sains Malaysia that fund us through the grant number: 1001.PMEKANIK.810301. We also would like to thank the Hospital Universiti Sains Malaysia that provides us with the test studies and perspective of the working conditions in the hospital during the pandemic.

\section{References}

[1] Ahad, Nor Aishah, Friday Zinzendoff Okwonu, and Pang Yik Siong. "COVID-19 Outbreak in Malaysia: Investigation on Fatality Cases." Journal of Advanced Research in Applied Sciences and Engineering Technology 20, no. 1 (2020): 1-10. https://doi.org/10.37934/araset.20.1.110

[2] COVID-19 Dashboard by the CSSE at Johns Hopkins University. Accessed on July 27, 2021. https://www.arcgis.com/apps/opsdashboard/index.html

[3] Chen, Huang-Chi, Mei-Hsing Chen, Chun-Wei Shen, Meng-Hsuan Hsieh, Lin-Kun Wu, Li-Chin Chen, Tsun-Jen Cheng, Ling-Sui Chen, Jong-Rung Tsai, and Shih-Huai Hsiao. "Secure Health Care Workers' Health and Safety Methodically During COVID-19 Epidemic in Taiwan." Asia Pacific Journal of Public Health 32, no. 8 (2020): 485-488. https://doi.org/10.1177/1010539520963629

[4] Schneider, Sandra, Brar Piening, Pauline Assina Nouri-Pasovsky, Anne Caroline Krüger, Petra Gastmeier, and Seven Johannes Sam Aghdassi. "SARS-Coronavirus-2 cases in healthcare workers may not regularly originate from patient care: lessons from a university hospital on the underestimated risk of healthcare worker to healthcare worker transmission." Antimicrobial Resistance \& Infection Control 9, no. 1 (2020): 1-7. https://doi.org/10.1186/s13756$\underline{020-00848-w}$

[5] Ran, Li, Xuyu Chen, Ying Wang, Wenwen Wu, Ling Zhang, and Xiaodong Tan. "Risk factors of healthcare workers with corona virus disease 2019: a retrospective cohort study in a designated hospital of Wuhan in China." Clinical Infectious Diseases 71-16 (2020): 2218-2221. https://doi.org/10.1093/cid/ciaa287

[6] Zhang, Shuai, Mengfei Guo, Feng Wu, Nian Xiong, Yanling Ma, Zhihui Wang, Limin Duan, Lan Chen, Haixia Ouyang, and Yang Jin. "Factors associated with asymptomatic infection in health-care workers with severe acute respiratory syndrome coronavirus 2 infection in Wuhan, China: a multicentre retrospective cohort study." Clinical Microbiology and Infection 26, no. 12 (2020): 1670-1675. https://doi.org/10.1016/i.cmi.2020.08.038

[7] Tong, Xin, Mingzhe Ning, Rui Huang, Bei Jia, Xiaomin Yan, Yali Xiong, Weihua Wu, Jiacheng Liu, Yuxin Chen, and Chao Wu. "Surveillance of SARS-CoV-2 infection among frontline health care workers in Wuhan during COVID-19 outbreak." Immunity, Inflammation and Disease 8, no. 4 (2020): 840-843. https://doi.org/10.1002/iid3.340

[8] Vimercati, Luigi, Luigi De Maria, Marco Quarato, Antonio Caputi, Pasquale Stefanizzi, Loreto Gesualdo, Giovanni Migliore et al. "COVID-19 hospital outbreaks: Protecting healthcare workers to protect frail patients. An Italian observational cohort study." International Journal of Infectious Diseases 102 (2021): 532-537. https://doi.org/10.1016/j.ijid.2020.10.098

[9] Cook, T. M., K. El-Boghdadly, B. McGuire, A. F. McNarry, A. Patel, and A. Higgs. "Consensus guidelines for managing the airway in patients with COVID-19: Guidelines from the Difficult Airway Society, the Association of Anaesthetists the Intensive Care Society, the Faculty of Intensive Care Medicine and the Royal College of Anaesthetists." Anaesthesia 75, no. 6 (2020): 785-799. https://doi.org/10.1111/anae.15054

[10] Chen, Xiangdong, Yanhong Liu, Yahong Gong, Xiangyang Guo, Mingzhang Zuo, Jun Li, Wenzhu Shi et al. "Perioperative management of patients infected with the novel coronavirus: recommendation from the Joint Task Force of the Chinese Society of Anesthesiology and the Chinese Association of Anesthesiologists." Anesthesiology 132, no. 6 (2020): 1307-1316. 
[11] Miller, Shelly L., Debanjan Mukherjee, Joseph Wilson, Nicholas Clements, and Cedric Steiner. "Implementing a negative pressure isolation space within a skilled nursing facility to control SARS-CoV-2 transmission." American journal of infection control 49, no. 4 (2021): 438-446. https://doi.org/10.1016/j.ajic.2020.09.014

[12] Al-Benna, Sammy. "Negative pressure rooms and COVID-19." Journal of Perioperative Practice 31, no. 1-2 (2021): 18-23. https://doi.org/10.1177/1750458920949453

[13] Huang, Wen-Nan, Mao-Song Zhuang, Tsun-Jen Cheng, and Shih-Huai Hsiao. "Simplest Way to Establish COVID-19 Quarantine Observation Wards Within 24 Hours." Asia Pacific Journal of Public Health 32, no. 6-7 (2020): $357-359$. https://doi.org/10.1177/1010539520947874

[14] Joseph, Bobby, and Merlyn Joseph. "The health of the healthcare workers." Indian journal of occupational and environmental medicine 20, no. 2 (2016): 71. https://doi.org/10.4103/0019-5278.197518

[15] Mukhopadhyay, Tapasyapreeti, Jay Relan, Arulselvi Subramanian, and Amit Lathwal. "Knowledge, attitude and practice of health care professionals on laboratory diagnosis of COVID-19." Journal of Family Medicine and Primary Care 10, no. 5 (2021): 1922. https://doi.org/10.4103/ifmpc.jfmpc 245920

[16] AstroCel II Low Pressure Drop Mini-Pleat Hepa/Ulpa Filter. AAF Flanders. Updated 2021. Accessed on August 1 2021. https://www.aafintl.com/-/media/files/aaf/commercial-and-industrial/us-products/hepa---ulpa/astrocellii/astrocel-ii prod mark broch afp-1-404.pdf

[17] Center for Disease Control and Prevention. Guidelines for Environmental Infection Control in Health-Care Facilities. Updated June, 2003. Accessed on August 1, 2021. https://www.cdc.gov/mmwr/preview/mmwrhtml/rr5210a1.htm 